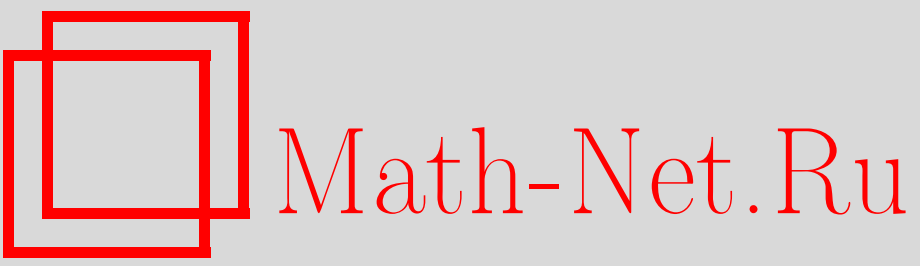

Л. А. Лютикова, Логический подход к нахождению выбросов в данных, Итоги науки и техн. Сер. Соврем. мат. и ее прил. Темат. обз., 2019, том 166, 49-56

DOI: https://doi.org/10.36535/0233-6723-2019-166-49-56

Использование Общероссийского математического портала Math-Net.Ru подразумевает, что вы прочитали и согласны с пользовательским соглашением

http://www.mathnet.ru/rus/agreement

Параметры загрузки:

IP: 54.162 .85 .209

26 апреля 2023 г., 12:50:03 


\title{
ЛОГИЧЕСКИЙ ПОДХОД К НАХОЖДЕНИЮ ВЫБРОСОВ В ДАННЫХ
}

\author{
(c) 2019 г. $\quad$ Л. А. ЛЮтИКОВА
}

\begin{abstract}
АннотАция. В работе рассмотрен логический подход к вопросам классификации данных и выявлению выбросов в них. Предложен алгоритм нахождения всех возможных классов для заданной предметной области, построенный на основе логической функции-классификатора. Проведен анализ этих классов и выявление наиболее выраженных закономерностей, по которым должна проводиться классификация.
\end{abstract}

Ключевъе слова: логические алгоритмы, выбросы, классификатор, информативное значение, весовые характеристики.

\section{LOGICAL APPROACH TO FINDING OUTLIERS IN DATA}

\section{(c) 2019 L. A. LYUTIKOVA}

\begin{abstract}
In this paper, we discuss a logical approach to classification of data and identification of outliers in them. We propose an algorithm for finding all possible classes for a given subject area based on the classifier logical function. We analyze these classes and identify the most pronounced patterns corresponding to the classification principles.
\end{abstract}

Keywords and phrases: logical algorithm, outlier, classifier, informative value, weight characteristic.

AMS Subject Classification: $68 \mathrm{~T} 27$

1. Введение. Проблема качества данных является на сегодняшний день одной из важнейших проблем, решаемых при построении интеллектуальных систем. Особенно остро данная проблема проявляется при построении обучающихся систем распознавания. Основным отличием обучающихся систем распознавания является построение классификатора в результате обучения. Процесс обучения заключается в последовательном предъявлении системе исходного множества объектов и корректировке классификатора в соответствии с этими объектами. Именно поэтому наличие искаженных данных способно существенно повлиять на конечную работу классификатора и снизить его эффективность. Искаженные данные будем считать выбросами. Причины появления в обучающей выборке искаженных данных могут быть самыми разнообразными: от сбоев в оборудовании и шумов до неверной интерпретации эксперта. Основным объединяющим свойством искаженных данных является то, что они не могут выступать в качестве эталонов обучающей выборки.

Поскольку невозможно избавиться от подобного рода данных, возникает задача их выявления для уменьшения влияния на процесс обучения.

В настоящие время существуют хорошо зарекомендовавшие себя методы выявления выбросов. K ним относятся статистические тесты, модельные тесты, итерационные методы, метрические методы, методы машинного обучения, изолирующие леса и т. д. (см. [1]).

В данной работе предлагается логический анализ данных на предмет присутствия в них выбросов и построение робастной процедуры, нечувствительной к их влиянию.

Работа выполнена при поддержке Российского фонда фундаментальных исследований (проект № 18-01-00050-а). 
2. Постановка задачи. Распознавание образов - это уже целый раздел теоретической информатики, разрабатывающий принципы и методы классификации и идентификации предметов, явлений, процессов, сигналов, ситуаций - всех тех объектов, которые могут быть описаны конечным набором некоторых признаков или свойств, характеризующих объект (см. $[2,6])$.

Описание объекта представляет собой $n$-мерный вектор, где $n$-число признаков, используемых для характеристики объекта, причем $j$-я координата этого вектора равна значению $j$-го признака, $j=1, \ldots, n$. В описании объекта допустимо отсутствие информации о значении того или иного признака.

Формальная постановка задачи распознавания образов такова.

Пусть $X=\left\{x_{1}, x_{2}, \ldots, x_{n}\right\}, x_{i} \in\left\{0,1, \ldots, k_{i}-1\right\}$, где $k_{i} \in[2, \ldots, N], N \in \mathbb{N}$, - множество признаков; $Y=\left\{y_{1}, y_{2}, \ldots, y_{m}\right\}$ - множество объектов, причем каждый объект $y_{i}$ характеризуется соответствующим набором признаков $x_{1}\left(y_{i}\right), \ldots, x_{n}\left(y_{i}\right): y_{i}=f\left(x_{1}\left(y_{i}\right), \ldots, x_{n}\left(y_{i}\right)\right)$. Иначе, пусть $X=\left\{x_{1}, x_{2}, \ldots, x_{n}\right\}$, где $x_{i} \in\left\{0,1, \ldots, k_{r}-1\right\}, k_{r} \in[2, \ldots, N], N \in Z$, - обрабатываемые входные данные $x_{i}=\left\{x_{1}\left(y_{i}\right), x_{2}\left(y_{i}\right), \ldots, x_{n}\left(y_{i}\right)\right\}, i=1, \ldots, n, y_{i} \in Y, Y=\left\{y_{1}, y_{2}, \ldots, y_{m}\right\}$-выходные данные:

Вид функции $Y=f(X)$ не задан.

$$
\left(\begin{array}{cccc}
x_{1}\left(y_{1}\right) & x_{2}\left(y_{1}\right) & \ldots & x_{n}\left(y_{1}\right) \\
x_{1}\left(y_{2}\right) & x_{2}\left(y_{2}\right) & \ldots & x_{n}\left(y_{2}\right) \\
\ldots & \ldots & \ldots & \ldots \\
x_{1}\left(y_{m}\right) & x_{2}\left(y_{m}\right) & \ldots & x_{n}\left(y_{m}\right)
\end{array}\right) \rightarrow\left(\begin{array}{c}
y_{1} \\
y_{2} \\
\ldots \\
y_{m}
\end{array}\right)
$$

Для восстановления этой функции по наблюдениям требуется определить качество данных. Надо заметить, что среди данных могут находиться те, которые могут существенно искажать работу функции классификатора. Как правило, подобные данные выявляются при помощи различных статистических методов, которые хорошо себя зарекомендовали при решении подобных задач и их дальнейшим удалением, либо построением робастных процедур, т.е. процедур, нечувствительным к выбросам.

Данные, с которыми приходится иметь дела при решении задач распознавания, как известно, являются неполными, неточными, неоднозначными. Однако получаемые решения должны соответствовать закономерностям, явно и неявно присутствующим в рассматриваемой предметной области. Логические методы могут достаточно хорошо проанализировать данные, выделить существенные и несущественные признаки, выявить минимальный набор правил, необходимый для того, чтобы полностью восстановить исходные закономерности. В результате можно получить более компактное и надежное представление исходной информации, обрабатывать которую надежней и быстрее.

Будем говорить, что построенная система правил является полной, если она обеспечивает вывод всех возможных решений исходной области.

Группу объектов, выделенных по определенному признаку (группе признаков), будем называть классом. Каждый объект - представитель некоторого класса однотипных объектов, для которых класс определяет общие свойства: состав и структуру данных.

Зависимость между объектом и характеризующими его признаками представим правилом продукции:

$$
\bigotimes_{j=1}^{m} x_{j}\left(y_{i}\right) \rightarrow P\left(y_{i}\right), \quad x_{j}\left(y_{i}\right) \in\{0,1, \ldots, k-1\}, \quad i=1, \ldots, l,
$$

где предикат $P\left(y_{i}\right)$ принимает значения

$$
P\left(y_{i}\right)= \begin{cases}1, & \text { если } y=y_{i} \\ 0, & \text { если } y \neq y_{i}\end{cases}
$$

или

$$
\bigvee_{i=1}^{n} \bar{x}_{i}\left(y_{j}\right) \vee P\left(y_{j}\right), \quad j \in[1, \ldots, m]
$$


Решающей функиией назовем конъюнкцию всех решающих правил

$$
\bigotimes_{j=1}^{m} x_{j}\left(y_{i}\right) \rightarrow P\left(y_{i}\right), \quad x_{j}\left(y_{i}\right) \in\{0,1, \ldots, k-1\}, \quad i=1, \ldots, l ;
$$

иными словами,

$$
f(X)=\bigotimes_{j=1}^{m}\left(\bigvee_{i=1}^{n} \bar{x}_{i} \vee P\left(y_{i}\right)\right) .
$$

Функцию (1) можно интерпретировать следующим образом. Если обучающую выборку, состоящую из $k$ элементов, описать булевой функцией

$$
F\left(x_{1}\left(y_{i}\right), \ldots, x_{n}\left(y_{i}\right), P^{\sigma}\left(y_{1}\right), \ldots, P^{\sigma}\left(y_{n}\right)\right), \quad \text { где } \quad P^{\sigma}\left(y_{i}\right)=\left\{\begin{array}{ll}
\overline{P\left(y_{i}\right)} & \text { при } \sigma=0, \\
P\left(y_{i}\right) & \text { при } \sigma \neq 0,
\end{array},\right.
$$

то данная функция на наборах $\left(x_{1}\left(y_{i}\right), \ldots, x_{n}\left(y_{i}\right), P^{\sigma}\left(y_{1}\right), \ldots, \overline{P^{\sigma}\left(y_{i}\right)}, \ldots, P^{\sigma}\left(y_{n}\right)\right)$ принимает значение «0», а на всех остальных наборах - значение «1», т.е. она допускает любые отношения между признаками и объектами, кроме отрицания объекта на множестве признаков, характеризующих этот объект в обучающей выборке.

Эта функция однозначно характеризует исходные данные, разбивает предметную область на все возможные классы, обладает свойствами модифицируемости, отвечает требованиям полноты, непротиворечивости в заданной области.

Данная функция обладает рядом свойств и особенностей; она практически строит базу знаний для заданной области данных. Все свойства функции (1) подробно рассмотрены в [6].

При раскрытии скобок сокращение соответствующих элементов проводится по следующим правилам:

(i) если некоторая переменная входит в ДНФ с одним знаком во всех дизъюнктах, то удаляем все дизъюнкты, содержащие эту переменную (данная переменная не информативна);

(ii) если в ДНФ имеется какой-либо однолитерный дизъюнкт $x_{i}^{j}$, то удаляем все дизъюнкты вида $x_{i}^{j} \&$ (правило поглощения).

Получим тупиковую дизъюнктивную форму относительно начальной; ее можно характеризовать как набор аксиом для рассматриваемых данных, из которых может быть найдена любая взаимосвязь между объектами и их характеристиками на заданной области (см. [4]).

Определение 1. Логическим описанием класса $K_{j}$ назовем дизъюнкт, содержащий некоторое множество предикатов, отражающих объекты обучающей выборки, и переменные, характеризующие признаки этих объектов.

Утверждение 1. Функиия

$$
f(X)=\bigotimes_{i=1}^{n}\left(\bigvee_{j=1}^{m} \bar{x}\left(y_{j}\right) \vee y_{j}\right), \quad x\left(y_{j}\right) \in[0,1,2], \quad y_{j} \in Y,
$$

полна на заданном множестве признаков.

Доказательство. Система функций $\{f(X)\}$ называется полной на заданном множестве, если для каждого набора признаков $X_{j} \in X$ можно сделать хотя бы один вывод $f\left(X_{j}\right)=y_{j}$. Функция при умножении:

$$
\bar{x}_{i}\left(y_{j}\right) \& \bar{x}_{i}\left(y_{l}\right)= \begin{cases}\bar{x}_{i}\left(y_{j}\right) & \text { при } \bar{x}_{i}\left(y_{j}\right)=\bar{x}_{i}\left(y_{l}\right), \\ 0 & \text { в противном случае. }\end{cases}
$$

Тогда

$$
y_{j} \bar{x}_{i}\left(y_{l}\right) \vee \bar{x}_{i}\left(y_{j}\right) \& \bar{x}_{i}\left(y_{l}\right)= \begin{cases}\bar{x}_{i}\left(y_{j}\right) & \text { при } \bar{x}_{i}\left(y_{j}\right)=\bar{x}_{i}\left(y_{l}\right), \\ y_{j} \& \bar{x}_{i}\left(y_{l}\right) & \text { в противном случае. }\end{cases}
$$


Если $\bar{x}_{i}\left(y_{j}\right) \neq \bar{x}_{i}\left(y_{l}\right)$, то $x_{i}\left(y_{j}\right) \in \bar{x}_{i}\left(y_{l}\right)$; дизъюнкт $y_{j} \& \bar{x}_{i}\left(y_{l}\right)$ идентифицирует объект $y_{j}$ по признаку $x_{i}\left(y_{l}\right)$. Подобные рассуждения можно провести по каждому из признаков. В результате можно утверждать, что для каждого набора признаков $X_{j} \in X$ можно сделать хотя бы один вывод $f\left(X_{j}\right)=y_{j}$. Утверждение доказано.

Теорема 1. Необходимым и достаточным условием принадлежсности оббекта, характеризуемого набором признаков $\left\{X_{j}\right\}, \kappa$ эталонному элементу или классу $K_{j}$ является выводимость следующих правил:

$$
P\left(y_{j}\right)=f_{1}\left(X_{j}\right), \quad P\left(K_{j}\right)=f_{2}\left(X_{j}\right) .
$$

Функция состоит из конечного числа дизъюнктов, часть из которых являются аксиомами; это те дизъюнкты, которые содержат минимальное число объектов в качестве сомножителей. Другая часть дизъюнктов является классами; это дизъюнкты, содержащие как можно больше объектов. Наконец, имеются дизъюнкты, которые не содержат объектов вообще и состоят только из переменных. Иначе говоря, $f(X)$ можно условно представить как дизъюнкцию трех функций:

$$
f(X)=f_{1}(X) \vee f_{2}(X) \vee f_{3}(X),
$$

где $f_{1}(X)$ - система аксиом, или индивидуальные признаки определенных объектов; $f_{2}(X)$ - множество классов исходной базы данных и $f_{3}(X)$ - множество настроечных элементов, которые не имеют значения для вывода, но имеют значение в случае поступления новой информации и модификации функции.

Функция $f_{2}(X)$ даёт картину разбиения базы данных на классы. Дизъюнкты, входящие в данную функцию, должны содержать как можно больше компонентов (объектов). Функция $f_{2}(X) \vee f_{1}(X)$ является объектной частью решающей функции. Функция $f_{3}(X)$ не имеет отношения ни к выводу, ни к совокупности классов, ни к идентификации объектов. Она состоит только из дизъюнктов, элементами которых являются переменные (признаки объектов); $f_{3}(X)$ является настроечной функцией, так как она предоставляет информацию о неиспользованных переменных или их определенных наборах, которые могут в дальнейшем стать основными идентифицирующими признаками для новых элементов множества $\{Y\}$. В силу этого $f_{3}(X)$ не играет роли для идентификации выбросов, и мы ее опустим в дальнейших рассуждениях (см. [3]).

3. Алгоритм моделирования объектной части решающей функции и построение классов. Для реализации объектной части решающей функции можно воспользоваться следующим алгоритмом:

(1) организуем двумерный массив с переменным количеством строк и количеством столбцов, записывая численные значения каждого признака;

(2) соблюдая порядок следования заданных объектов, каждый элемент ставим в соответствующую ему строку и в соответствующий численному значению каждого характеризующего признака столбец:

\begin{tabular}{|c|c|c|c|c|c|c|c|c|c|}
\hline $0_{1}$ & $1_{1}$ & $k_{1}-1$ & $0_{2}$ & $1_{2}$ & $k_{2}-1$ & $\ldots$ & $0_{n}$ & $1_{n}$ & $k_{n}-1$ \\
\hline & $y_{1}$ & & & & & $\ldots$ & & & $y_{1}$ \\
\hline & $y_{2}$ & & & & & $\ldots$ & & $y_{2}$ & \\
\hline$\ldots$ & $\ldots$ & $\ldots$ & $\ldots$ & $\ldots$ & $\ldots$ & $\ldots$ & $\ldots$ & $\ldots$ & $\ldots$ \\
\hline$y_{m}$ & & & & $y_{m}$ & & $\ldots$ & & & $y_{m}$ \\
\hline
\end{tabular}

(3) проверяем каждый столбец заданного массива; если в столбце более одного элемента, то вычеркиваем эти элементы из данных строк и заносим их в следующую строку в тот же 
столбец:

\begin{tabular}{|c|c|c|c|c|c|c|c|c|c|}
\hline $0_{1}$ & $1_{1}$ & $k_{1}-1$ & $0_{2}$ & $1_{2}$ & $k_{2}-1$ & $\ldots$ & $0_{n}$ & $1_{n}$ & $k_{n}-1$ \\
\hline & $y_{1}$ & & & & & $\ldots$ & & & $y_{1}$ \\
\hline & $y_{2}$ & & & & & $\ldots$ & & $y_{2}$ & \\
\hline$\ldots$ & $\ldots$ & $\ldots$ & $\ldots$ & $\ldots$ & $\ldots$ & $\ldots$ & $\ldots$ & $\ldots$ & $\ldots$ \\
\hline$y_{m}$ & & & & $y_{m}$ & & $\ldots$ & & & $y_{\text {mR }}$ \\
\hline & & & & & & & & & $y_{1} y_{m}$ \\
\hline
\end{tabular}

эти элементы образуют класс по данному значению переменной;

(4) проверяем строки; если в строке, соответствующей какому-либо объекту остались переменные, то объект идентифицируется именно по этим переменным. Совокупность отдельных объектов и их индивидуальных признаков будут являться аксиомами для заданной области. Строки, у которых несколько объектов в одном столбце, демонстрируют классы, которые возможно получить исследуя данную предметную область.

Пример. Рассмотрим набор данных, характеризуемый таблицей

\begin{tabular}{|c|c|c|c|c|}
\hline$x_{1}$ & $x_{2}$ & $x_{3}$ & $x_{4}$ & $y$ \\
\hline 0 & 1 & 2 & 0 & $a$ \\
\hline 0 & 2 & 1 & 0 & $b$ \\
\hline 1 & 0 & 1 & 1 & $c$ \\
\hline 1 & 1 & 1 & 1 & $d$ \\
\hline 0 & 0 & 0 & 2 & $e$ \\
\hline
\end{tabular}

Не строя всю функцию-классификатор целиком, построим для наглядности только ее объектную часть в соответствии с описанным выше алгоритмом:

\begin{tabular}{|c|c|c|c|c|c|c|c|c|c|c|c|}
\hline \multicolumn{2}{|c|}{$x_{1}$} & \multicolumn{3}{|c|}{$x_{2}$} & \multicolumn{3}{|c|}{$x_{3}$} & \multicolumn{3}{|c|}{$x_{4}$} & \multirow[t]{2}{*}{$K$ (классы) } \\
\hline 0 & 1 & 0 & 1 & 2 & 0 & 1 & 2 & 0 & 1 & 2 & \\
\hline d & & & $\alpha$ & & & & $a$ & $\alpha$ & & & \\
\hline \& & & & & $b$ & & k & & 8 & & & \\
\hline$\partial k$ & & & & & & & & $a b$ & & & $a b x_{1}^{0} x_{4}^{0}$ \\
\hline & $£$ & $£$ & & & & $£$ & & & 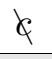 & & \\
\hline & & & & & & $\partial \alpha$ & & & & & $b c x_{3}^{1}$ \\
\hline & $d$ & & $d$ & & & $d$ & & & $d$ & & \\
\hline & & & & & & $b c d$ & & & & & $b c d x_{3}^{1}$ \\
\hline & $c d$ & & & & & & & & $c d$ & & $c d x_{1}^{1} x_{4}^{1} x_{3}^{1}$ \\
\hline & & & $a d$ & & & & & & & & $a d x_{2}^{1}$ \\
\hline k & & k & & & $e$ & & & & & $e$ & \\
\hline$a b e$ & & & & & & & & & & & $a b e x_{1}^{0}$ \\
\hline & ce & & & & & & & & & & $c e x_{2}^{0}$ \\
\hline
\end{tabular}

После работы алгоритма мы получили все возможные классы на рассматриваемых данных:

$$
\left\{a b x_{1}^{0} x_{4}^{0}, b c d x_{3}^{1}, c d x_{1}^{1} x_{4}^{1} x_{3}^{1}, a d x_{2}^{1}, a b e x_{1}^{0}, c e x_{2}^{0}, b x_{2}^{2}, a x_{3}^{2}, e x_{3}^{0} x_{4}^{2}\right\} .
$$


Объектная часть, описанной ранее функции будет выглядеть следующим образом:

$$
f_{2}(X)=a b x_{1}^{0} x_{4}^{0} \vee b c d x_{3}^{1} \vee c d x_{1}^{1} x_{4}^{1} x_{3}^{1} \vee a d x_{2}^{1} \vee a b e x_{1}^{0} \vee c e x_{2}^{0} \vee b x_{2}^{2} \vee a x_{3}^{2} \vee e x_{3}^{0} x_{4}^{2}
$$

Определение 2. Число объектов, объединенных в класс по совокупности признаков, назовем объектным весом класса; обозначение $v_{\text {об }}$.

Определение 3. Число признаков, объединяющих в класс определенное количество объектов, назовем признаковым весом класса; обозначение $v_{\text {приз }}$

Так,

$$
v_{\text {об }}\left(b c d x_{3}^{1}\right)=3, \quad v_{\text {приз }}\left(c d x_{1}^{1} x_{4}^{1} x_{3}^{1}\right)=3 .
$$

4. Логический метод обнаружения выбросов. В работе претендентами на выбросы будем называть объекты, которые не входят в основные классы. Для рассмотренного выше примера это $b x_{2}^{2}, a x_{3}^{2}$ и $е x_{3}^{0} x_{4}^{2}$.

Введем понятие информативности класса, представленного конъюнкциями.

Класс - это множество объектов и характеризующих их признаков. Для информативной характеристики совокупности количества объектов класса и количества определяющих этот класс признаков введем следующую величину - информативный вес:

$$
w_{i}=\frac{n_{i}}{n}+\frac{m_{i}}{m}
$$

где $n_{i}$ - число признаков, определяющих класс $K_{i}, n$-общее число признаков, $m_{i}$ - число объектов попадающих в класс $K_{i}, m$ - общее число объектов исследуемой предметной области.

Для разбиения предметной области на классы, а так же определения в ней выбросов относительно полученного разбиения предлагается следующий алгоритм.

1. Определить информационный вес каждой конъюнкции, полученной в результате работы предложенного выше алгоритма.

2. Выбрать конъюнкции $K_{i}$ с наибольшим весом $w_{i}$.

3. Вычеркнуть все конъюнкции, содержащие входящие в класс $K_{i}$ объекты.

4. Перейти к шагу 2.

5. После того как остались классы, не пересекающиеся по объектам, найти число классов

$$
L=\bmod \sum_{i} w_{i}
$$

6. Если существует такой $K_{j}$, что $w_{j}$ не влияет на $L$, то $K_{i}$ является выбросом для данной классификации.

Для данного примера $n=4$ (четыре признака), $m=5$ (пять объектов).

Шаг 1. Подсчитаем информативные веса у каждой конъюнкции, найдем наибольший вес и вычеркнем все конъюнкции, содержащие элементы с наибольшим весом:

\begin{tabular}{|c|c|}
\hline$K$ (класс, конъюкция $)$ & $w$ \\
\hline$a b x_{1}^{0} x_{4}^{0}$ & $18 / 20$ \\
\hline$b c d x_{3}^{1}$ & $17 / 20($ содержит $c d)$ \\
\hline$c d x_{1}^{1} x_{4}^{1} x_{3}^{1}$ & $23 / 20-$ максимальное значение веса \\
\hline$a d x_{2}^{1}$ & $13 / 20($ содержит $d)$ \\
\hline$a b e x_{1}^{0}$ & $17 / 20$ \\
\hline$c е x_{2}^{0}$ & $13 / 20($ содержит $c)$ \\
\hline$b x_{2}^{2}$ & $9 / 20$ \\
\hline$a x_{3}^{2}$ & $9 / 20$ \\
\hline$e x_{3}^{0} x_{4}^{2}$ & $14 / 20$ \\
\hline
\end{tabular}


Шаг 2. Из оставшихся конъюнкций найдем конъюнкцию с наибольшим весом и проведем те же операции:

\begin{tabular}{|c|c|}
\hline$K$ (класс, конъюкция) & $w$ \\
\hline$a b x_{1}^{0} x_{4}^{0}$ & $18 / 20$ максимальное значение веса \\
\hline$a b e x_{1}^{0}$ & $17 / 20$ содержит $a b$ \\
\hline$b x_{2}^{2}$ & $9 / 20$ содержит $b$ \\
\hline$a x_{2}^{2}$ & $9 / 20$ содержит $a$ \\
\hline$e x_{3}^{0} x_{4}^{2}$ & $14 / 20$ \\
\hline
\end{tabular}

На втором шаге получили класс $a b x_{1}^{0} x_{4}^{0}$ с весом $w_{a b}=18 / 20$ и $е x_{3}^{0} x_{4}^{2}$ с $w_{e}=14 / 20$;

$$
L=\bmod \left(w_{c d}+w_{a b}+w_{e}\right)=\bmod \left(\frac{23}{20}+\frac{18}{20}+\frac{14}{20}\right)=2 .
$$

В данном примере для введенной нами весовой информативности мы получаем будет два класса: это классы с наибольшими весами $c d x_{1}^{1} x_{4}^{1} x_{3}^{1}$ и $a b x_{1}^{0} x_{4}^{0}$, причем

$$
L=\bmod \left(w_{c d}+w_{a b}\right)=\bmod \left(\frac{23}{20}+\frac{18}{20}\right)=2,
$$

а также выброс $е x_{3}^{0} x_{4}^{2}$ для полученной классификации. Как видно, информативный вес объекта не влияет на количество классов, поэтому его можно трактовать как выброс.

Поэтому можно говорить, что представленные данные поддаются следующей классификации: $c d x_{1}^{1} x_{4}^{1} x_{3}^{1}$ и $a b x_{1}^{0} x_{4}^{0}$

\begin{tabular}{|c|c|c|c|c|}
\hline$x_{1}$ & $x_{2}$ & $x_{3}$ & $x_{4}$ & $y$ \\
\hline $\mathbf{0}$ & 1 & 2 & $\mathbf{0}$ & $\mathbf{a}$ \\
\hline $\mathbf{0}$ & 2 & 1 & $\mathbf{0}$ & $\mathbf{b}$ \\
\hline$\underline{1}$ & 0 & $\underline{1}$ & $\underline{1}$ & $\underline{\mathbf{c}}$ \\
\hline$\underline{1}$ & 1 & $\underline{1}$ & $\underline{1}$ & $\underline{\mathbf{d}}$ \\
\hline 0 & 0 & 0 & 2 & $e$ \\
\hline
\end{tabular}

Объект е является выбросом для выбранной классификации.

Утверждение 2. Обгект $y_{i} \in Y$ в множестве $Y=\left\{y_{1}, y_{2}, \ldots, y_{m}\right\}$ классифицируемых объектов является выбросом в рамках модели, в которой величина информативного веса представлена как $w_{i}=n_{i} / n+m_{i} / m$, где $n_{i}$ - число признаков, определяющих класс $K_{i}, n$-общее число признаков, $m_{i}$ - число обгектов попадающих в класс $K_{i}, m$-общее число обгектов исследуемой предметной области, если

$$
L=\bmod \sum_{i} w_{i}=\bmod \left(\sum_{j} w_{j}-w_{i}\right),
$$

где $i$-номер класса, полученный после логической классификации заданной предметной облаcmu.

\section{СПИСОК ЛИТЕРАТУРЫ}

1. Дъяконов А. Г., Головина А. М. Выявление аномалий в работе механизмов методами машинного обучения. Аналитика и управление данными в областях с интенсивным использованием данных// XIX Междунар. конф. DAMDID/RCDL'2017 (Москва, 10-13 октября 2017 г.). - М., 2017. - С. 469-476.

2. Журавлёв Ю. И. Об алгебраическом подходе к решению задач распознавания или классификации// Пробл. киберн. - 1978. - 33. - С. 5-68.

3. Лютикова Л. А., Шматова Е. В. Анализ и синтез алгоритмов распознавания образов с использованием переменнозначной логики// Информ. технол. -2016. - 22, № 4. - С. 292-297. 


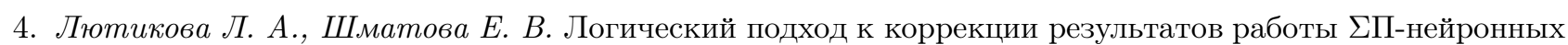
сетей// Информ. технол. - 2018. - 24, № 2. - С. 110-116.

5. Шибзухов 3. М. О принципе минимизации эмпирического риска на основе усредняющих агрегирующих функций// Докл. РАН. - 2017. - 476, № 5. - С. 495-499.

6. Флах П. Машинное обучение. Наука и исскуство построения алгоритмов, которые извлекают знания из данных. - М.: МДК Пресс, 2015.

Лютикова Лариса Адольфовна

Институт прикладной математики и автоматизации,

Кабардино-Балкарский научный центр Российской академии наук, Нальчик, Россия

E-mail: lylarisa@yandex.ru 